\title{
Modeling the effect of strong magnetic field on n-type MOSFET in strong inversion
}

\author{
D.-V. Nguyen, L. Werling, C. Po, N. Dumas, M. \\ Madec, W. Uhring, L. Hébrard \\ ICube - Université de Strasbourg / CNRS \\ 23, rue du Lœss - Strasbourg, France \\ duc-vinh.nguyen@etu.unistra.fr
}

\begin{abstract}
This paper presents an approach to model the effect of magnetic field on the electrical behavior of MOS transistor. It is shown that an out-of-plane magnetic field $B$ induces a reduction of the channel conductivity, and that this reduction is proportional to the square of $B$ and the square of the carrier mobility. Providing an accurate model of the mobility versus the gate to source and drain to source voltages in all working conditions of the transistor, the effect of $B$ on the MOS transistor can be accurately modeled. Experimental data in strong inversion and linear regime are in very good agreement with the proposed model.
\end{abstract}

Keywords—MOS transistors, strong magnetic field, modeling

\section{INTRODUCTION}

Nuclear Magnetic Resonance (NMR) is widely used in analytical chemistry, and its application to Magnetic Resonance Imaging (MRI) for medical diagnostic is now of routine use. Due to the inherent low sensitivity of NMR, engineers target the development of NMR/MRI systems at very high magnetic field. Indeed, the Signal-to-Noise Ratio (SNR) of NMR experiments scales with the static magnetic field $B_{0}$ used to magnetize the sample. This SNR scaling ranges from $B_{0}$ to $B_{0}^{7 / 4}$, depending on the sample properties (conducting or not) and on the loss in the antenna used to pick-up the Free Induction Decay (FID) signal [1]. Another way to increase the FID signal is to use an antenna with a filling factor close to $100 \%$, i.e. whose sensing volume is the same as the sample volume [2]. This condition is ideally obtained with micro-coils [3], which in addition helps in having a good homogeneity of $B_{0}$, leading to high resolution NMR spectrum [4]. The last source of loss is the preamplifying electronics which is generally deported, the antenna being connected to the preamplifier through a transmission line [5]. So, in order to get the best sensitivity, it is of primary importance to have a very high $B_{0}$ field, and to use a micro-antenna with a cointegrated electronics, or with a preamplifier placed very close to the antenna. The electronics has thus to withstand this strong $B_{0}$ field. In analytical NMR, field up to $28.2 \mathrm{~T}$ are in use [1]. Although the most widespread magnets for biomedical and preclinical MRI and MRS (MR-Spectroscopy) on animal models are $7 \mathrm{~T}$ and $14.1 \mathrm{~T}$, magnets up to $21.1 \mathrm{~T}$ are used in research [1]. Finally human-body MRI scanner are only approved for $1.5 \mathrm{~T}$ and $3 \mathrm{~T}$, however in brain research, static fields up to $10.5 \mathrm{~T}$ are in use [1].

\author{
L. Fakri-Bouchet ${ }^{1}$, J. Pascal ${ }^{2}$, Y. Wadghiri ${ }^{3}$ \\ ${ }^{1}$ ISA - Université Claude Bernard Lyon 1 / CNRS \\ 5 rue de la Doua, Villeurbanne, France \\ ${ }^{2}$ University of Applied Sciences and Arts Northwestern \\ Switzerland FHNW, Muttenz, Switzerland \\ ${ }^{3}$ NYU School of Medicine, $66011^{\text {st }}$ Ave, New-York, NY
}

10016 (USA)

Twenty years ago, a few publications have reported parasitic effects on the electrical characteristics of Field Effect Transistors [6] [7]. However no research has been performed on the topic, certainly because the applications of such very high fields are limited. With the advent of ultra-high field NMR/MRI systems [1], and the trend to place the electronics inside the field, close to the sample, studying the effect of strong field on the electrical characteristics of MOS transistors becomes relevant. We have recently published a paper showing that under an outof-plane magnetic field of $B=7 \mathrm{~T}$, i.e. a 7T field perpendicular to the plane of the chip, the current and the transconductance of NMOS transistors exhibiting a high $W / L$ ratio may be reduced by up to $7 \%$ [8]. In addition, this current and transconductance reduction is proportional to $\tan \left(\mu_{n}^{2} \cdot B^{2}\right)$, where $\mu_{n}$ is the mobility of electrons in the MOS channel. In silicon, where $\mu_{n}$ is low (typically between 300 and $400 \mathrm{~cm}^{2} / \mathrm{V} \cdot \mathrm{s}$ ), for field below $15 \mathrm{~T}, \tan \left(\mu_{n}^{2} \cdot B^{2}\right) \approx \mu_{n}^{2} \cdot B^{2}$. As a consequence a $28 \%$ reduction of the MOS transconductance is expected under $14 \mathrm{~T}$, which may be very challenging in terms of circuit design. We have also shown that for transistors with a high $W / L($ small $L / W)$, which is common in circuit design, the current reduction comes from the deflection of the current lines inside the channel due to the Lorentz force $(-q \cdot v \times B)$ acting on the carriers [8]. Here $q$ is the elementary electric charge, $v$ is the drift velocity of electrons, and the symbol $\times$ denotes the vector cross product. Although our first experimental setup was very basic, we were able to show in [8] that the current reduction only depends on the out-of-plane component of the magnetic field, and that, as expected, the tilt angle $\mu_{n}^{2} \cdot B^{2}$ depends on the gate to source voltage through the electron mobility reduction with $V_{G S}$, i.e. with the transverse electric field. A very simple model was also proposed where the length of the transistor is changed as $L$. $\cos \left(\mu_{n}^{2} \cdot B^{2}\right)$ in order to take the current line tilt into account. However, as shown below, this model is too much simplified. In this paper we push forward our study with an improved experimental setup, and we pave the way towards the development of a transistor model able to help the designer in taking into account the effect of strong magnetic fields on integrated circuits. Focus is put on transistors with a high $W / L$ ratio since they are the most affected by magnetic field. A brief discussion on long transistors, i.e. with a small $W / L$ is also given.

Section II settles the theoretical background necessary to guide the experiment and to develop the model. Section III describes the improvement in the experimental setup and the 
measurement results. Section IV discusses these results and the limitation of the model. It is followed by a conclusion.

\section{THEORYTICAL BACKGROUND}

\section{A. Geometrical magnetoresistance}

At least in linear regime, the MOS channel can be seen as a conventional linear resistance, where the density of carriers $n$ is roughly constant (for a given $V_{G S}$ ), as well as the longitudinal electric field $E_{l}$, that is given by $V_{D S} / L$ where $V_{D S}$ is the drain to source voltage. When a magnetic field $B$ is applied, the moving electrons feels the Laplace force:

$$
F_{L}=-q \cdot E_{l}-q \cdot v \times B
$$

The energy they acquire with $F_{L}$ is lost in the silicon lattice through scattering, and the resulting equilibrium leads to an average electron velocity proportional to $F_{L}$ :

$$
v=\frac{\mu_{n}}{q} \cdot F_{L}=-\mu_{n} \cdot E_{l}-\mu_{n} \cdot v \times B
$$

where $\mu_{n}$ is the above mentioned electron mobility. The current density is thus:

$$
j=n \cdot q \cdot v=n \cdot q \cdot \mu_{n} \cdot E_{l}-\mu_{n} \cdot j \times B
$$

Using the relation $(U \times V) \bullet W=(U \bullet V) \cdot V-(V \bullet W) \cdot U$, where $\bullet$ stands for the scalar product, and $U, V$, and $W$ are three vectors, expression (1) can be solved under perpendicular magnetic field, i.e. $E_{l} \bullet B=0$, leading to:

$$
j=\frac{\sigma_{n} \cdot E_{l}+\mu_{n} \cdot \sigma_{n} \cdot\left(E_{l} \times B\right)}{1+\mu_{n}^{2} \cdot B^{2}}
$$

where $\sigma_{n}=n \cdot q \cdot \mu_{n}$ is the silicon conductivity.

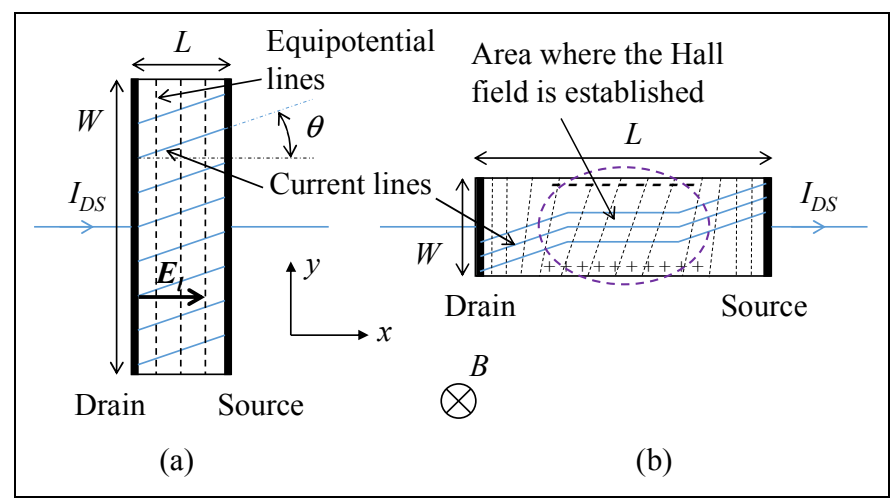

Fig.1. a) short transistor, b) long transistor, under $B$.

As seen in figure 1-a, for a short channel, i.e. a transistor with a small $L / W$ ratio, the drift electric field in the channel, $E_{l}$, remains along the $x$ axis, i.e. perpendicular to the equipotential lines formed by the source and drain that are assumed as ohmic contacts (see figure 1-a for axis definition). Expression (2) shows that the drift current is no longer along $E_{l}$. It is tilt by the angle $\theta$, where $\tan (\theta)=\mu_{n} \cdot B$ and can be decomposed as a longitudinal component, $j_{x}$, and a perpendicular component, $j_{y}$ :

$$
j_{x}=\frac{\sigma_{n} \cdot E_{l}}{1+\mu_{n}^{2} \cdot B^{2}} \text { and } j_{y}=\frac{\mu_{n} \cdot \sigma_{n} \cdot\left(E_{l} \times B\right)}{1+\mu_{n}^{2} \cdot B^{2}}
$$

For a short channel the electrons which tend to accumulate on the lateral side, due to $j_{y}$, flow immediately back through the source and drain ohmic contacts. On the contrary for long transistors, i.e. when $L / W$ is high, in the center of the channel the resistive path for the electrons to flow back through the source and drain is too high, and electrons accumulate on one side of the channel where a negative surface charge appears, while the other side exhibits a deficit of electrons, i.e. a positive surface charge. This brings an electric field along $y$, the Hall field, and an electric force opposite to the Lorentz force. Finally, both forces are balanced, and the current lines remain along $x$ in the center of the channel (see figure 1-b) while the electric field $E_{l}$ is no longer aligned with $\mathrm{x}$. A Hall voltage appears between the lateral sides of the MOS channel [9].

The only current density component leading to a drainsource current $I_{D S}$ is $j_{x}$. So, for a small $L / W$ transistor, the expression of $j_{x}$ in equation (3) shows that under a magnetic field, the channel conductivity, respectively the carrier mobility, is reduced to an effective conductivity $\sigma_{n}(B)$, respectively an effective mobility $\mu_{n}(B)$, given by:

$$
\sigma_{n}(B)=\frac{\sigma_{n}}{1+\mu_{n}^{2} \cdot B^{2}} \text { and } \mu_{n}(B)=\frac{\mu_{n}}{1+\mu_{n}^{2} \cdot B^{2}}
$$

As a consequence, for a small $L / W$ transistor, the transconductance parameter $K P_{n}=\mu_{n} \cdot C_{o x}$ has to be changed in:

$$
K P_{n}(B)=\frac{\mu_{n}}{1+\mu_{n}^{2} \cdot B^{2}} \cdot C_{o x}=\mu_{n}(B) \cdot C_{o x}
$$

in the expression of the $I_{D S}$ current, where the parameter $C_{o x}$ is the gate capacitance per unit area.

For long transistors, i.e. with a high $L / W$, rewriting equation (1) as:

$$
E_{l}=\frac{j}{\sigma_{n}}+\frac{\mu_{n}}{\sigma_{n}} \cdot j \times B
$$

shows that in the center of the channel, where $j$ is aligned along $x$, the electric field is now the sum of a $x$-component given by $E_{x}=j / \sigma_{n}$ and a $y$-component given by $E_{y}=\left(\mu_{n} / \sigma_{n}\right) \cdot j \times B$. As a consequence, in the center, the channel conductivity is unaffected by the magnetic field and remains equal to $\sigma_{n}$. Of course, close to the drain and the source, this is no longer valid since the current lines are deflected (see figure 1-b).

To summarize, the deflection of the current lines leads to an effective mobility of the carriers in the calculation of $I_{D S}$ given by $\mu_{n} /\left(1+\tan ^{2} \theta\right)$ where $\theta$ is the deflection angle. For small $L / W$ transistors, $\theta$ remains constant, equal to $\mu_{n} \cdot B$ (we assume $B<15 \mathrm{~T}$ ), and $I_{D S}$ is simply reduced by the reduction factor (RF):

$$
R F=1 /\left(1+\mu_{n}^{2} \cdot B^{2}\right) \approx 1-\mu_{n}^{2} \cdot B^{2}
$$

For high $L / W$ transistor, $\theta$ varies along the channel and the computation of $I_{D S}$ is more complex. It will not be addressed in this paper.

It is worth to notice that our first model [8], where we just modified the length of the transistor, i.e. $L$ changed in $L \cdot \cos \theta$, is too much approximate since it leads to a current reduction factor of $1-\mu_{n}^{2} \cdot B^{2} / 2$. 
However, whatever the model, the Relative Reduction Factor, $R R F$, is proportional to $\mu_{n}^{2} \cdot B^{2}$ :

$$
R R F=\frac{I_{D S}(B)-I_{D S}}{I_{D S}} \approx-\mu_{n}^{2} \cdot B^{2}
$$

It thus depends on the carrier mobility which is well known to depend on $V_{G S}$ and $V_{D S}$. The effect of magnetic field on MOS transistors is thus depending on the transistor biasing, and in order to derive a compact model of the transistor behavior, we have to consider the mobility dependence on $V_{G S}$ and $V_{D S}$.

\section{B. Electron mobility}

In a MOS channel, electron mobility is known to depend on the longitudinal electric field, i.e. on $V_{D S}$, and on the transverse electric field, i.e. on $V_{G S}$.

- Mobility dependence on $V_{D S}$

The longitudinal electric field $E_{l}$ leads to the electron drift from source to drain. Under high field, the electron drift velocity, $v=\mu_{n} \cdot E_{l}$ saturates, and the mobility is reduced. In silicon, a good model for the dependence of $\mu_{n}$ on $E_{l}$ is given by [10]:

$$
\mu_{n}\left(E_{l}\right)=\frac{\mu_{0}}{\left(1+\left(\mu_{0} \cdot E_{l} / v_{S A T}\right)^{\beta}\right)^{1 / \beta}}
$$

where $\mu_{0}$ is the mobility at low field (from 300 to $400 \mathrm{~cm}^{2} / \mathrm{V} \cdot \mathrm{s}$ ), $v_{S A T}$ is the saturation velocity, and $\beta$ a fitting parameter. Typical values for $\beta$ and $v_{S A T}$ in a n-type channel are [11]:

$$
\beta=2 \text { and } 4 \cdot 10^{6} \mathrm{~cm} / \mathrm{s}<v_{S A T}<8.5 \cdot 10^{6} \mathrm{~cm} / \mathrm{s}
$$

For short transistors, i.e. with a low $L / W, E_{l}$ can be approximated by $E_{l}=V_{D S} / L$. So, in our model, we will use the following expression for $\mu_{n}\left(V_{D S}\right)$ :

$$
\mu_{n}\left(V_{D S}\right)=\frac{\mu_{0}}{\sqrt{1+\left(\mu_{0} \cdot V_{D S} /\left(v_{S A T} \cdot L\right)\right)^{2}}}
$$

where $\mu_{0}$ and $v_{S A T}$ will be determined from the experimental measurements.

\section{- Mobility dependence on $V_{G S}$}

The transverse electric field $E_{t}$, leading to the creation of the electron channel, pushes the electrons towards the $\mathrm{SiO}_{2} / \mathrm{Si}$ interface where the carriers feel enhanced scattering effects due to interface states and surface roughness [11]. As a consequence, their mobility is reduced when $V_{G S}$ increases.

$E_{t}$ can be expressed as [11]:

$$
E_{t}=\frac{Q_{B}\left(V_{\sigma}\right)}{\varepsilon_{s i}} \cdot\left(1+\frac{V_{G S}-V_{T H}}{V_{o v}}\right) \text { with } V_{o v}=2 \cdot Q_{B}\left(V_{\sigma}\right) / C_{o x}
$$

where $V_{T H}$ is the threshold voltage of the transistor, and $V_{o v}$ stands for the overdrive voltage above which the degradation of the mobility by the transverse field dominates. The depletion charge $Q_{B}$ below the channel depends on the surface voltage $V_{\sigma}$ at the $\mathrm{SiO}_{2} / \mathrm{Si}$ interface. However when the transistor is in linear regime, $V_{\sigma}$ is roughly constant, and thus $V_{o v}$. Under strong transverse field, many experiments show that $\log \left(\mu_{n}\right)$ decreases linearly with $\log \left(V_{G S}-V_{T H}\right)$. It means a mobility dependence on $V_{G S}-V_{T H}$ proportional to $1 /\left(\left(V_{G S}-V_{T H}\right) / V_{o v}\right)^{\delta}$ where $\delta$ is a fitting parameter that depends on the interface states density and substrate doping [11]. However, when $V_{G S}-V_{T H}$ is low, typically below $1 \mathrm{~V}$ from our own measurements, this simple dependence on $V_{G S}$ cannot fit the measurements since at small $V_{G S}-V_{T H}$ the mobility becomes roughly constant. We thus propose to use an equation similar to equation (4) to model the mobility dependence on $V_{G S}$ :

$$
\mu_{n}\left(V_{G S}\right)=\frac{\mu_{0}}{\left(1+\left(\left(V_{G S}-V_{T H}\right) / V_{o v}\right)^{a}\right)^{b}}
$$

where $\mu_{0}, V_{o v}, a$, and $b$ are determined from the experiments.

\section{EXPERIMENTS}

\section{A. Experimental setup}

As for our first measurements, the chip was placed in a 7T Bruker BioSpec 70/30 MRI scanner [8]. However, the setup was drastically improved. First the chip was no longer encapsulated in a package but directly bonded on a small PCB, and no BNC connector, nor coaxial cable were used. This choice minimizes the influence of the setup on the direction of the magnetic field seen by the chip. In addition a plexilass holder was etched by laser cutting to be able to precisely place the chip inside the MRI bore (see figure 2). The remaining measurement procedure is the same and all details may be found in [8].

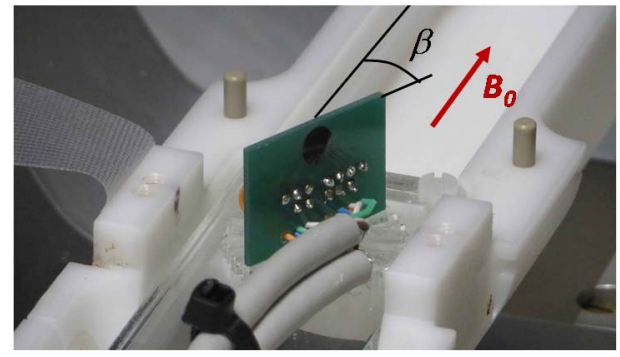

Fig. 2. The chip is placed in its plexiglass holder and tilt by $\beta=30^{\circ}$ with respect to the $B_{0}=7 \mathrm{~T}$ field direction.

\section{B. Measurements}

For sake of space, the results presented here are focused on a two-finger NMOS transistor whose $L / W$ is $0.7 / 20$, i.e. each finger sizes $0.7 / 10$ and features a small $L / W$. As in [8], $I_{D S^{-}} V_{G S}$ characteristics were recorded for a varying $V_{D S}$, from $10 \mathrm{mV}$ to $500 \mathrm{mV}$ by step of $20 \mathrm{mV}$. The transconductance $\mathrm{d} I_{D S} / \mathrm{d} V_{G S}$ was computed versus $V_{G S}$, and the slope $\alpha=\mu_{n} \cdot C_{o x} \cdot W / L \cdot V_{D S}$ was determined at $V_{G S O}$ for which the transconductance is maximum. The threshold voltage was also determined [8]. Finally, the slope $\alpha$ was computed for $V_{G S}-V_{T H}$ varying from $150 \mathrm{mV}$ to $1.1 \mathrm{~V}$, i.e. under strong inversion condition, by step of $20 \mathrm{mV}$. Then the RRF was calculated as:

$$
R R F=\frac{\alpha(B)-\alpha}{\alpha} \text { i.e. } R F F=\frac{\mu_{n}(B)-\mu_{n}}{\mu_{n}} \approx-\mu_{n}^{2} \cdot B^{2}
$$

Figure 3 shows $R R F$ at $V_{G S O}$, i.e. at maximum transconductance, versus $V_{D S}$ for three orientations of the chip, $\beta=90^{\circ}, 60^{\circ}$, and $30^{\circ}$ where $\beta$ is defined in figure 2 . Using equation (4), a fitting of the data at $\beta=90^{\circ}$ leads to the parameters $\mu_{0}=371 \mathrm{~cm}^{2} / \mathrm{V} \cdot \mathrm{s}$ and $v_{S A T}=4.9 \cdot 10^{6} \mathrm{~cm} / \mathrm{s}$, in good accordance with [11]. Then the same parameters were used 
to compute the expression $-\mu_{n}^{2} \cdot B^{2} \cdot \sin ^{2}(B)$ that should fit the data recorded when the chip is placed at $\beta=60^{\circ}$ and $30^{\circ}$. As seen in figure 3 , the model is in very good agreement with the experiments, confirming that the transistor is only sensitive to the magnetic field component perpendicular to the chip plane.

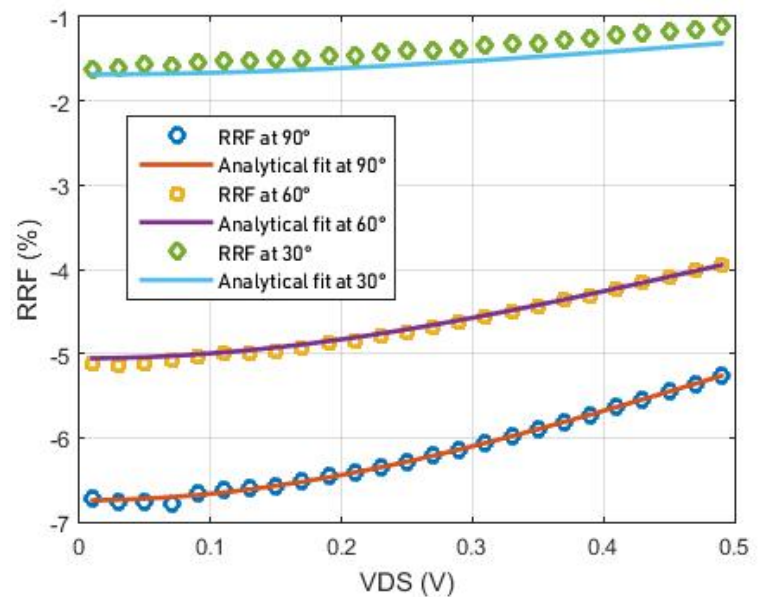

Fig. 3. $R R F$ at maximum transconductance versus $V_{D S}$ for three $\beta$ angles.

Figure 4 shows $R R F$ for the chip at $\beta=90^{\circ}$ computed versus $V_{G S}-V_{T H}$ at $V_{D S}=10 \mathrm{mV}$, as well as its fitting by the analytical expression $-\mu_{n}^{2} \cdot B^{2}$ where equation (6) is used for $\mu_{n}\left(V_{G S}\right)$. Here again, the model is in very good agreement with the experimental data.

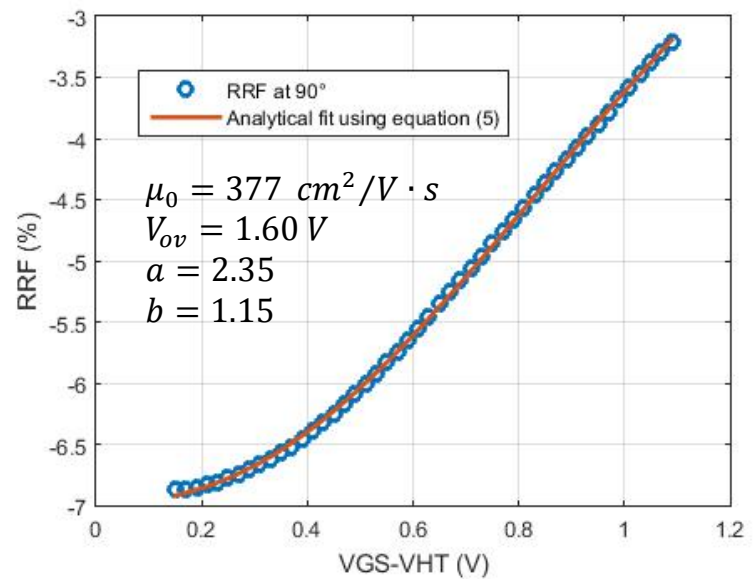

Fig. 4. $R R F$ versus $V_{G S}-V_{T H}$ at $V_{D S}=10 \mathrm{mV}$

\section{DISCUSSION AND MODEL LIMITATION}

This study shows that under a magnetic field $B$ perpendicular to the chip, the main parameters of the circuit designers, i.e. the biasing current $I_{D S}$ and the transconductance $g_{m}$ feature a relative reduction equal to $-\mu_{n}^{2} \cdot B^{2}$. It is also shown that the dependence of $\mu_{n}$ on $V_{G S}$ and $V_{D S}$ has to be taken into account to accurately model the magnetic field effect. As long as the transistor remains in linear regime and strong inversion, the proposed model, where $\mu_{n}$ is given by the product of equation (5) and (6) allows an accurate prediction of the magnetic field effect for short transistors.
In saturation, expression (5) may be used provided that the saturation voltage $V_{D S s} \approx V_{G S}-V_{T H}$ is substituted for $V_{D S}$. However expression (6) is no longer valid since the surface voltage $V_{\sigma}$ is no longer constant along the channel, and $V_{o v}$ depends on $V_{D S s}$. Finally, for long transistor, i.e. exhibiting a high $L / W, R R F$ is reduced and a correction factor has to be introduced in order the model the current line deflection which varies along the channel.

\section{CONCLUSION}

This paper has presented our first step towards the modeling of the effect of strong magnetic field on the electrical behavior of MOS transistors. It is shown that for short transistors, the biasing current and the transconductance are reduced by a factor of $-\mu_{n}^{2} \cdot B^{2}$. Although only valid when the transistor is in linear regime and in strong inversion, the proposed approach may be used to model the transistor behavior in saturation and/or in moderate and weak inversion, as soon as an appropriate model for the mobility is used. For long transistors, a geometrical factor depending on $L / W$ has to be introduced. These developments will be the subject of future work.

\section{ACKNOWLEDGMENT}

The authors acknowledge the French ANR agency for its financial support through the ANR-16-CE19-0002-01 project.

\section{REFERENCES}

[1] E. Moser, E. Laistier, F. Schmitt, and G. Kontaxis, "Ulta-High Field NMR and MRI - The Role of Magnet Technology to Increase Sensitivity and Specificity”, Frontiers in Physics, vol. 5, Article 33, August 2017.

[2] D. Hoult, R. Richards, "The signal to noise ratio of the nuclear magnetic resonance experiment", Journal of Magnetic Resonance, 1976, pp. 24-71

[3] R. M. Fratila and A. H. Velders, "Small-Volume Nuclear Magnetic Resonance Spectroscopy", Annual Review of Analytical Chemistry, vol. 4, 2011, pp. 227-249

[4] J. E. Stocker, T. L. Peck, A. G. Webb, M. Feng, and R. L. Magin, "Nanoliter Volume, High-Resolution NMR Microspectroscopy Using a 60- $\mu \mathrm{m}$ Planar Microcoil”, IEEE Trans. On Biomedical Engineering, vol. 44, $\mathrm{N}^{\circ} 11$, November 1997, pp. 1122-1127

[5] Andrew G Webb, Magnetic Resonance Technology: Hardware and System Component Design, Royal society of Chemistry, 2016

[6] J. R. Bodart, and al., "The effect of high magnetic fields on junction field effect transistor device performance", Review of Scientific Instruments, 1998, pp. 319-320

[7] P. Phothimat, M. Awipi, "Effect of High Magnetic Field on Transistor Characteristics With Applications to SEU Testing", Proc. of IEEE Southeastcon'98, 1998, pp. 338-339

[8] L. Hébrard, and al., "On the influence of strong magnetic field on MOS transistors", Proc. of IEEE ICECS 2016, 11-14 Dec., 2016, pp. 564-657

[9] R. S. Popovic, "Hall Effect Devices", Second Ed., Institute of Physics Publishing, 2004

[10] J. B. Roldan, and al., "The dependance of the electron mobility on the longitudinal electric field I MOSFETs", Semiconductor Science and Technology, vol. 12, 1997, pp. 321-330

[11] A. Modelli and S. Manzini, "High-field drift velocity of electrons in silicon inersion layers", Solid-State Electronics, vol. 31, $\mathrm{n}^{\circ} 1,1988$, pp. 99-104

[12] S. C. Sun and J. D. Plummer, "Electron mobility in inversion and accumulation layers on thermally oxidized silicon surfaces", IEEE Trans. On Electron Devices, vol. 27, $\mathrm{n}^{\circ}$ 8, August 1980, pp. 1497-150 